\title{
Multimodal Artifact Metrics and its Applicability Experiments
}

\author{
Masaki Fujikawa a, ${ }^{\mathrm{a},}$, Kouki Jitsukawaa, Shingo Fuchi ${ }^{\mathrm{b}}$ \\ aKogakuin University, 1-24-2 Nishi-shinjuku, Shinjuku, Tokyo 163-8677, JAPAN \\ ${ }^{b}$ Aoyama Gakuin University, 5-10-1 Fuchinobe, Chuo-ku, Kanagawa 252-5258, JAPAN \\ *Corresponding Author: masaki.fujikawa.jp@ieee.org
}

\begin{abstract}
In this paper, we propose multimodal artifact metrics. We came up with this idea based on multimodal identification metrics in biometrics and is expected to verify the artifact's authenticity with high accuracy by using more than two characteristic information with different physical characteristics extracted from the artifact. In this technique, counterfeiting of copied products seems to be more difficult even for the manufacturers who are producing the genuine products. In order to explore the feasibility of this idea, we present the results of two experiments: One is making samples made of conductive polymer and filler with optical characteristic. Another one is extracting two types of characteristic information from each sample. Extracted information (resistance and IR image) were different for each specimen, as we estimated.
\end{abstract}

Keywords: multimodal artifact metrics, characteristic information, verification of authenticity.

\section{Introduction}

\subsection{Background and target of our study}

Although the use of artifact metrics is a technique for verifying the authenticity of the artifacts produced daily by manufacturers, its concept is the same as that of biometrics. In artifact metrics, authenticity is verified using characteristic information extracted from an individual artifact. Similarly, biometrics identifies a person using bio-information extracted from an individual.

It can be said that the word "artifact metrics" stems from a word "biometrics." In biometrics, single modal techniques (methods that use a single type of bio-information to identify individuals) have been prevalent so far; however, multimodal techniques (methods that use multiple types of bio-information to identify individuals) have become common in recent years in order to identify a person with high accuracy and strengthen the robustness of the technique to impersonation attacks. In fact, a technical report on multimodal identification systems was published by $\mathrm{ISO}^{(1)}$, and in India, faces, fingerprints, and iris information are used to identify individuals in the national identification number project ${ }^{(2)}$.

Nowadays, a massive number of copied products that mimic genuine ones have been distributed. Hence, techniques for verifying the authenticity of artifacts with high accuracy and making counterfeiting more difficult are becoming increasingly necessary in manufacturing industries. Therefore, in this paper, we propose a technique that uses "multimodal artifact metrics," which are based on multimodal biometrics. This technique gives artifacts multiple types of characteristic information with different physical characteristics and can verify the authenticity of each artifact using this information.

This paper describes the following contents: In section 2 , we describe our technique's concept, prerequisites, and the artifacts targeted in this paper. In section 3 , we introduce the way of producing artifacts using our technique, extraction of characteristic information, and the method of verifying authenticity of each artifact. In section 4, we explain our experiments of verifying the effectiveness of our method and show its results. Authors describe the considerations for practical use in section 5 and conclude this paper in section 6 .

\subsection{Overview of artifact metrics}

In this section, we describe how characteristic information extracted from artifacts is used in artifact metrics. In artifact metrics, an artifact's unique characteristic information is extracted using sensing devices. This information is embedded in the artifact using the 
simple methods of adding materials (fillers) with one physical characteristic during the manufacturing process. The particles of the added fillers are distributed randomly and non-uniformly and fixed in the artifact. Their degree of distribution reflects the characteristic information. Table 1 shows the physical characteristics of fillers added in the manufacturing process and the characteristic information extracted from them.

Fig. 1 shows an overview of the proposed system that uses the artifact metrics. In this system, characteristic information is extracted from each artifact before it is shipped and the information is stored in a secure database. To verify the authenticity of an artifact, the system extracts the characteristic information from it and compares this information with the registered feature information from the secure database. These processes are almost the same as those of biometrics.

\subsection{Related studies}

In this section, authors introduce related studies in order to clarify our study's position.

Table 1. Physical features and extracted characteristics information

\begin{tabular}{c|l}
\hline $\begin{array}{c}\text { Physical } \\
\text { characteristics }\end{array}$ & \multicolumn{1}{c}{ Extracted feature information } \\
\hline $\begin{array}{c}\text { Optical } \\
\text { characteristics }\end{array}$ & $\begin{array}{l}\text { Particles' optical characteristics (reflection, } \\
\text { transmission, infraction, and fluorescence) and their } \\
\text { degree of distribution reflect the characteristic } \\
\text { information, which is extracted by sensors that can } \\
\text { detect light intensity }\end{array}$ \\
\hline $\begin{array}{c}\text { Magnetic } \\
\text { characteristics }\end{array}$ & $\begin{array}{l}\text { Particles' magnetic characteristics (attraction and } \\
\text { repulsive force) and their degree of distribution } \\
\text { reflect the characteristic information, which is } \\
\text { extracted by sensors that can detect a change in } \\
\text { magnetism. }\end{array}$ \\
\hline $\begin{array}{c}\text { Electrical } \\
\text { characteristics }\end{array}$ & $\begin{array}{l}\text { Particles' electric characteristics (electrical charge) } \\
\text { and the degree of distribution reflect the } \\
\text { characteristic information, which is extracted by } \\
\text { sensors that can detect the quantity of electric charge. }\end{array}$ \\
\hline $\begin{array}{c}\text { Vibration } \\
\text { characteristics }\end{array}$ & $\begin{array}{l}\text { Particles' vibration characteristics (sonic waves) and } \\
\text { the degree of distribution reflect the characteristic } \\
\text { information, which is extracted by sensors that can } \\
\text { detect sonic waves. }\end{array}$ \\
\hline
\end{tabular}

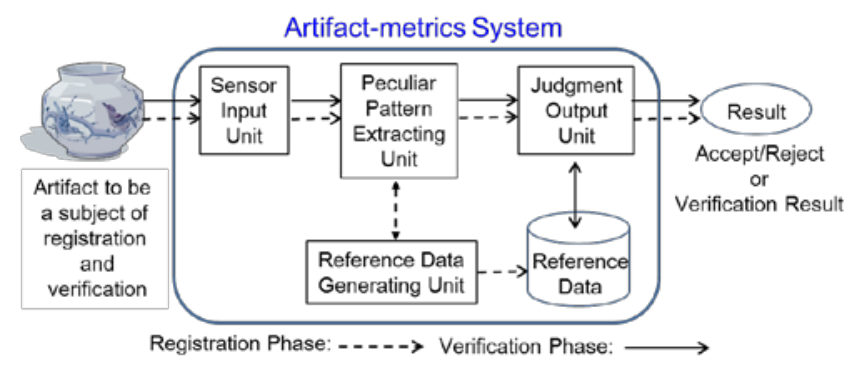

Fig. 1. Artifact metrics system.
Characteristic information extracted from artifacts can be changed depending on the environmental circumstances during extraction (such as temperature, humidity, and position of the artifacts relative to the sensing devices). However, even in such situations, the artifact metric system should be able to verify authenticity stably and correctly based on the strong correlation between the characteristic information registered in the database and the information features extracted during verification.

There is an approach to increase the types of characteristic information in order to find strong correlations between both information types. A method was proposed to extract two characteristic information from a filler with one physical feature ${ }^{(3)}$. In this method, a filler with an optical feature (glass phosphor powder) is mixed with paint and glaze and adhered onto the surface of porcelain in order to bond the particles of the filler onto the ceramics during the firing process. There is a difference between the density of light-emitting ions and the particle diameters that depends on the observation points. The $\operatorname{method}^{(3)}$ utilizes the difference between the distributions of the light-emitting spectrum and light-emitting intensity that is caused by this difference and uses these two distributions as characteristic information.

\section{Multimodal artifact metrics}

\subsection{Concept}

The idea for the technique proposed in this paper (multimodal artifact metrics) is based on multimodal identification in biometrics. However, unlike previous study $^{(3)}$, as described in Section 1.3, this technique gives an artifact two or more physical characteristics and extracts two or more types of characteristic information from them.

The main difference between our method and that of ${ }^{(3)}$ (i.e., the advantage of our method) is that it is able to increase the types of characteristic information that can be extracted from the artifact. This is because the previous study gives the artifact only one physical characteristic so that clearly fewer types of characteristic information can be extracted from the artifact, whereas our method gives the artifact two or more physical features.

Our method has two contributions to artifact metrics technology. One is the ability to verify the authenticity stably and correctly (as we mentioned in the previous section), as our method can find a strong correlation between the characteristic information registered in a 
database and the feature information extracted from the artifact by increasing the types of characteristic information contained in artifact. Another one is to heighten the difficulty of counterfeiting for forgers, as the number of characteristic information contained in genuine products is increased.

\subsection{Targeted artifact(application to synthetic resin products)}

Although we are still investigating artifacts that are appropriate for multimodal metrics, we focus here on artifacts made from synthetic resin as one application in order to verify the applicability of our method.

We have two reasons for focusing on this type of artifact. One is that such artifacts are common in our daily life. In general, synthetic resin is easy to form and resists acid and alkaline corrosion. Furthermore, it is used to add features and characteristics to products in order to make them fit for use. Although it has the reputation of being non-conductive, flammable, and non-biodegradable, new types of synthetic resin with electro-conductive, flame-retardant, and biodegradable features have been developed. It can also be recycled.

Another reason is the existence of copied synthetic resin products. Table 2 categorizes synthetic resin products. Products for which the existence of copied products have been confirmed are underlined and the reference given. (In Table 2, products are categorized into two groups, and the definition, purpose of use, and specific name of the synthetic resin are indicated.) Thus far, the existence of copied products such as bank cards, watches, and automobile parts have been confirmed. We predict that security devices such as SIM cards and hardware tokens will be copied in the near future.

\subsection{Requirements}

We propose a basic method by preparing two or more fillers and adding them to artifact during manufacturing, where each filler has one physical characteristic, to easily to give an artifact two or more physical characteristics. The advantage of this method is its simplicity when it comes to adding filler into the artifact. However, as shown in Fig. 2, several drawbacks such as the decrease in moldability, decline in strength, and increase in monetary cost of fillers should be considered ${ }^{(9)}$ if the amount of fillers are increased. Although the recommended amount of filler is not clearly indicated $^{(9)}$, it is desirable to add as little filler into an artifact as possible from the viewpoint of moldability, stren-
Table 2. Categorization of synthetic resin products.

\begin{tabular}{|c|c|}
\hline \multirow{3}{*}{ Type 1} & $\begin{array}{l}\text { Definition: } \\
\text { The majority of the artifact is formed of synthetic resin. }\end{array}$ \\
\hline & 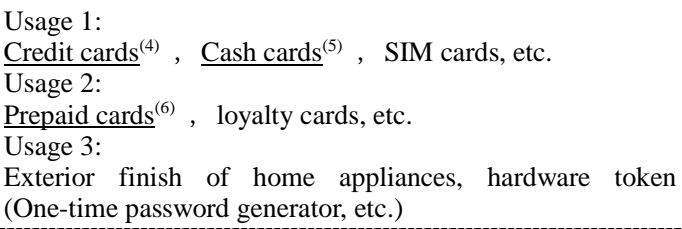 \\
\hline & $\begin{array}{l}\text { Material: } \\
\text { PVC is used for Usage } 1 \text {, PET is used for Usage 2, and ABS } \\
\text { is used for Usage } 3 \text {. }\end{array}$ \\
\hline \multirow{3}{*}{ Type 2} & $\begin{array}{l}\text { Definition: } \\
\text { The surface of the artifact is coated or painted by synthetic } \\
\text { resin. }\end{array}$ \\
\hline & $\begin{array}{l}\text { Usage 1: } \\
\text { Products made of ABS or polycarbonate without a baked } \\
\text { finish (e.g., wrist watches }{ }^{(7)} \text { and spectacle frames for } \\
\text { eyeglasses). This finish requires more than } 150^{\circ} \mathrm{C} \text {. } \\
\text { Usage 2: } \\
\text { Products made of aluminum or brass without a baked finish } \\
\text { (e.g., cast aluminum wheels }{ }^{(8)} \text { ). }\end{array}$ \\
\hline & $\begin{array}{l}\text { Material: } \\
\text { Acrylate resin, urethane resin, fluorine resin, or epoxy resin } \\
\text { is used for coating or painting in both usages. }\end{array}$ \\
\hline
\end{tabular}

gth, and economic cost. As described in Section 2.2, there are two types of resin (conductive and non-conductive synthetic resin). In either case, we conclude that we should not add $\mathrm{N}$ or more types of fillers in order to give $\mathrm{N}$ or more physical characteristics to an artifact. This means that a technique is needed that can give $\mathrm{N}$ or more physical characteristics to an artifact while reducing the number of fillers.

Next, the synthetic resin and filler added are required to have a low risk of irritating human skin, as users often come into contact with synthetic resin products, as shown in Table 2. Hence, in this paper, we define two requirements, as shown below:

Requirement 1: Although more than $\mathrm{N}$ or more physical characteristics can be added to synthetic resin, the number of fillers should be kept low.

Requirement 2: Synthetic resin and the filler added should not be a human skin irritant.

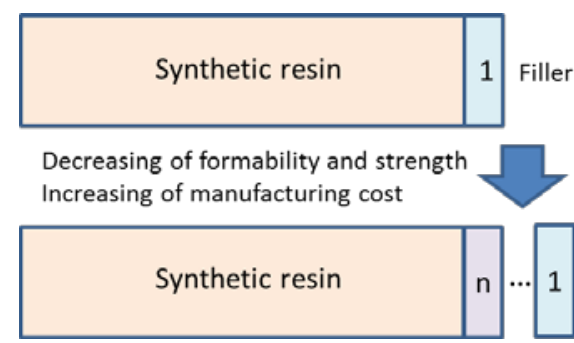

Fig. 2. Relationship between the number of fillers and the strength, formability, and monetary cost. 


\subsection{Preconditions}

We set the prerequisites shown below in order to clarify our discussion.

Condition 1: As described in Section 2.2, electro-conductive and non-conductive types of resin exist. In this study, as a first step, we focus on the former type and verify the applicability of multimodal artifact metrics on it. In concrete terms, we use a conductive polymer, as it is easy to handle, obtain, and is not a human skin irritant (fulfilling Property 2). In the near future, we plan to verify the applicability of the proposed technique on non-conductive resin

Condition 2: In multimodal artifact metrics, we give two or more physical characteristics to each artifact and extract two or more types of characteristic information from it. In this paper, as a first step, we give two physical characteristics to samples made of conductive polymer and extract two information features from them.

Condition 3: The goal of this paper is to determine whether we can apply multimodal artifact metrics to samples made of synthetic resin (conductive polymer). Hence, the implementation of multimodal artifact metrics (the construction of an artifact metric system and its evaluation) is not within the scope of this paper.

\section{Approach (application to conductive polymer)}

In this section, we describe the method to give two physical characteristics to samples made of conductive polymer and the way to extract two feature information from them.

\subsection{Overview of conductive polymer}

Conductive polymer is a type of synthetic resin with low electrical resistance. It is more flexible than ITO (Indium Tin Oxide), is used to form transparent electrodes, and has a high transparency (a thin film made of this polymer does not seriously degrade the base material's transparency). For this reason, it is used to form transparent electrodes as an alternative to ITO and as a flexible and transparent paint to prevent charging on an optical film.

Non-conductive synthetic resins such as PVC, PET, and ABS are used to make artifacts with various three-dimensional shapes. In contrast, conductive polymer is formed on the surface of an artifact as a thin film to give the artifact conductivity or as electric wiring for transmitting electrical signals or power.

\subsection{Making samples and forming information features}

Conductive polymer allows electrical current to flow easily and is mainly formed as a thin film. Hence, we attempted to make thin film samples onto a base material and added two physical characteristics to them. This polymer already has an electrical characteristic (low resistance), so we should consider how to add another characteristic to the samples and a method to embed the information features in each sample while taking advantage of the electrical feature of the resin (i.e., how to generate two types of information non-uniformly and randomly).

In the approach in this study, we employed a non-conductive powder with an optical characteristic (with a grain size of several micrometers) as filler and propose a method to form a thin film onto the base material using the mixture of conductive polymer and a small amount of filler. In this way, the connection between the particles of this polymer is variable because they are spread randomly and non-uniformly when the thin film is formed. Hence, we can extract electrical resistance as an information feature because it is reflected by the above connection (see Fig. 3). In addition, the reflection, transmission, and inflection of the light caused by filler particles and degree of filler powder dispersion are non-uniformly generated in the thin film. Hence, they can be observed from the intensity of light passing through the film and extracted as an information feature by taking an image of it using a camera. Specifically, one type of filler is added so that we can embed two types of characteristic information while reducing the amount and types of filler used.

Among the two information features obtained using the above approach, images can be obtained without contact; however, in order to obtain electrical resistance, probes should be touched to the samples. As the thin film formed onto the base material is soft, there is a risk of breaking/damaging the film by probes while measuring resistance.

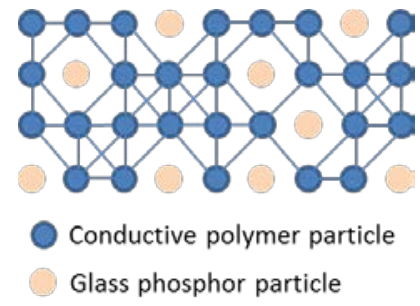

Fig. 3. Image of the density of bounded molecules. 


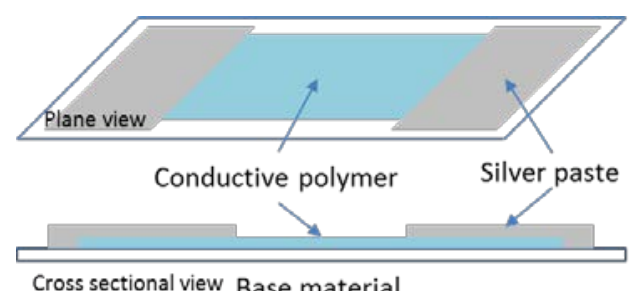

Cross sectional view Base material

Fig. 4. Sample construction.
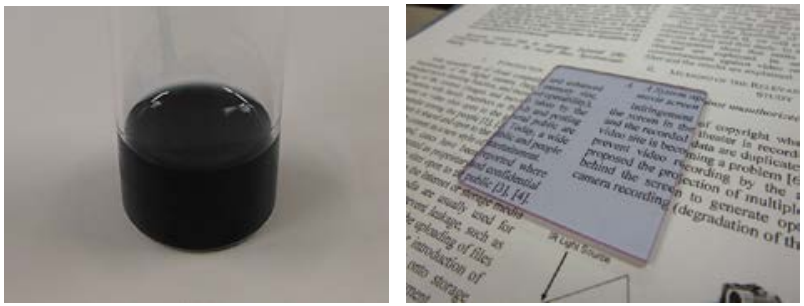

Fig. 5. Conductive polymer (left) and glass phosphor (right).

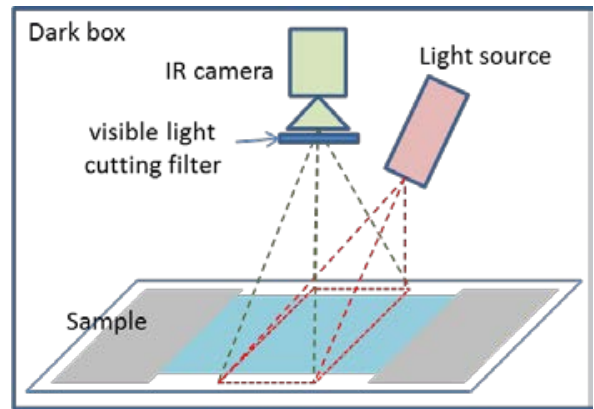

Fig. 6. Obtaining infrared images.

For this reason, we cover both ends of the thin film with silver paste (room-temperature curable type), as shown in Fig. 4 to form electrodes for contact with the probes in order to avoid breaking or damaging the film.

\subsection{Materials for making samples}

As described in previous section, we used conductive polymer (see Fig. 5, left) and a non-conductor with optical characteristics to make the samples. We used glass phosphor that we generated for the latter material (see Fig. 5 , right). We selected this polymer as the conductive synthetic resin not only because of its characteristic of allowing electrical current to flow easily. For this conductive polymer, the surface resistivity of the thin film (or its visible light transmittance) can be adjusted by changing its thickness. Hence, the density of the filler particle in the thin film affects its electric resistance. In general, in each sample, areas of sparse filler particles (dense polymer particles) and areas of dense filler particles (sparse polymer particles) are spontaneously generated so that they can be extracted as information features (or specific electrical resistances).

Furthermore, we have other reasons for selecting a conductive polymer: the surface resistivity of the thin film formed onto the base material does not change when a physical force is applied (flexion) and the conductivity is less likely to be changed by temperature ${ }^{(10)}$. This is because synthetic resin products are often subjected to physical force and exposed to wide range of temperatures. (In fact, conductive polymer is used as the material for electrolytic capacitors in order to gain stable equivalent series resistance (ESR) and impedance over a wide range of temperatures.)

Glass phosphor was selected for its features such as nonconductivity, low risk of irritating human skin, and infrared light emission under optical excitement ${ }^{(3)}$. (1) Infrared light emission from filler particles; (2) reflection, transmission, and inflection of infrared light; and (3) density of particles can be expressed as the intensity of infrared light, so infrared light images can be used to extract the information features.

Currently, glass phosphor cannot be produced on a large scale. Hence, in our experiments described in Section 4, we tried to find the appropriate amount of glass phosphor for adding to the polymer by conducting elementary tests with commercial phosphor. It has similar features as it emits a green light under optical excitation. In the later experiments, we used samples made from glass phosphor.

\subsection{Extraction of information features}

In this section, we describe how to extract characteristic information from samples. The conductive polymer we use has a surface resistivity (sheet resistance) of approximately $100 \Omega$ /square with $86 \%$ visible light transmittance and approximately $700 \Omega$ /square with $92 \%$ visible light transmittance. Although it is conductive, it does not need to have extra low surface resistivity (the samples' resistance will increase as filler is added to the conductive polymer). Hence, we adopted two-terminal measurement method for our samples instead of the four-terminal sensing method that is used for specimens with extremely low resistance. We fixed the distance between the terminals (electrodes) when measuring the resistance of the samples and confirmed the difference in resistance for each sample.

Next, we describe the collection of the infrared images. As shown in Fig. 6, an infrared camera faces the sample and we irradiate light onto the surface of the sample. As the 
particles of glass phosphor emit infrared light by optical excitation, we capture the scene with the infrared camera. We then confirm the difference of each image taken of the samples.

\subsection{Verification of authenticity}

Similar to other techniques proposed for artifact metrics so far, authenticity verification is done by calculating the degree of similarity between the information features registered in a secure database beforehand and those extracted during verification. When the value exceeds a threshold level, the objects are seemed to be genuine products.

As described in Section 2.4, the implementation of multimodal artifact metrics is out of the scope of this paper because this paper aims to give two physical characteristics to samples made of conductive polymer and extract two different information features from the samples. Hence, in this paper, we refrain from describing how to set the threshold level for calculating the degree of similarity. However, if we implement our technique, we should carefully calculate the degree of similarity in order to avoid incorrectly determining genuine products as copied products and vice versa ${ }^{(3)}$.

\section{Experiments}

\subsection{Appropriate amount of filler}

In order to determine the appropriate amount of glass phosphor, we verified whether the thin film was successfully formed onto the surface of the base material using a mixture of conductive polymer and commercial phosphor powder. We made four mixtures (conductive polymer combined with $5 \%, 10 \%, 20 \%$, and $40 \%$ glass phosphor powder by weight and attempted to form a thin film onto the surface of a clear base material. In this experiment, a thin blue film was formed from the $5 \%$ and $10 \%$ mixtures, but the other mixtures could not form a thin film and the existence of the phosphor powder was clearly visible (see Fig. 7). We believe that the number of conductive polymer particles needed to form the thin film was decreased because of the existence of a large number of phosphor particles.

Next, we cut the base material with the thin film (made of $5 \%$ and $10 \%$ commercial phosphor mixture) into strips 1 $\mathrm{cm}$ wide and painted silver paste onto both the edge of thin film in order to form terminals (electrodes). The area betw-
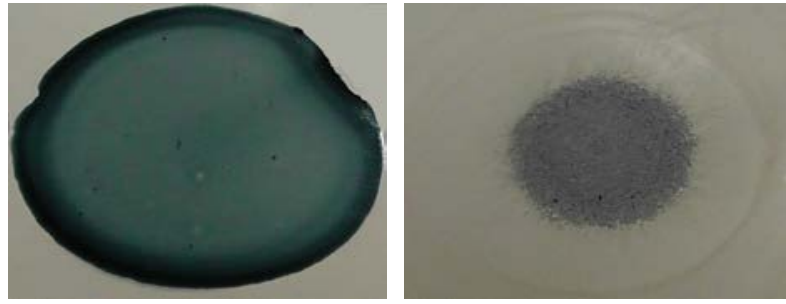

Fig. 7. Formed thin films (left: 5\%, right: 20\%).

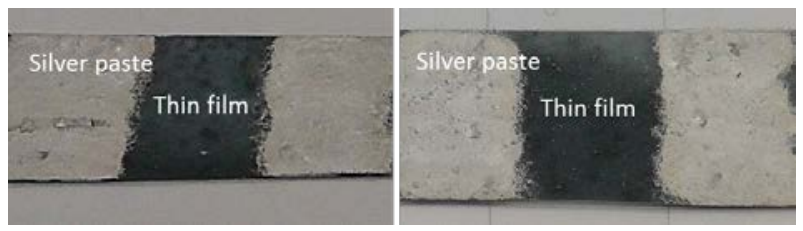

Fig. 8. Samples with commercial phosphor (left: 5\%, right: $10 \%)$.

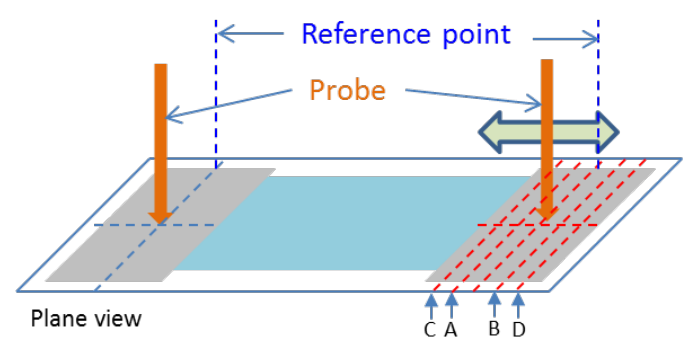

Fig. 9. Resistance measurement.

een the two terminals was about $1 \mathrm{~cm}$ square. We made 20 samples from each thin film (a total of 40 samples). Fig. 8 shows samples from each density.

As commercial phosphor emits light under optical excitation similarly to glass phosphor and the particles are distributed randomly in each sample, it is obvious that we can obtain different images from each sample based on the results of ${ }^{(3)}$. Thus, we measured the resistance of each sample in order to verify our hypothesis using the following method (as shown in Fig. 9).

(1) We touched the left probe to the reference point (center of the terminal).

(2) We touched the right probe to the reference point (0 $\mathrm{mm})$, points A \& B ( $\pm 2 \mathrm{~mm}$ from the reference point), and points $C \& D( \pm 4 \mathrm{~mm}$ from the reference point) and measured the resistance at each point.

Figs. 10 and 11 show the resistance of each sample. The vertical axis indicates the resistance, the horizontal axis indicates the distance from the reference point $(0 \mathrm{~mm})$, and the colored lines indicate the resistance of each sample. We find that the samples' resistances are clearly different and higher phosphor density leads to higher resistance. Hence, we determined that $5 \%$ glass phosphor should be added to the conductive polymer. In the rest of our paper, we 
describe the measurement of the resistance and camera imaging of the infrared images for samples made up of conductive resin with $5 \%$ glass phosphor.

\subsection{Extraction of information features (resistance)}

Example sample images made of conductive polymer and 5\% glass phosphor shown in Fig. 12 (we focused on areas the indicated by the yellow rectangles for the IR images). We made 14 samples using the method described in the previous section. Figure. 13 shows the resistance of the samples. Obviously, each sample has a different resistance.

\subsection{Extraction of information features (infrared light images)}

We took infrared light images from each sample using the method shown in Fig. 6. In order to avoid disturbance by ambient light, we used a dark box and set the equipment (light source, camera, and sample) into it. We irradiated the sample with light with a peak wavelength of $980 \mathrm{~nm}$ to excite the glass phosphor particles existing in the thin film and took photos of the film.

Infrared images of the samples shown in Fig. 12 are

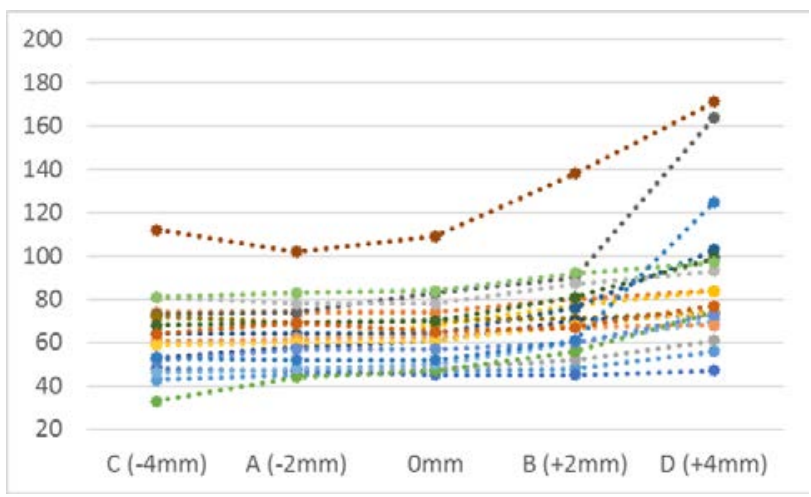

Fig. 10. Sample resistances (5\% glass phosphor by weight).

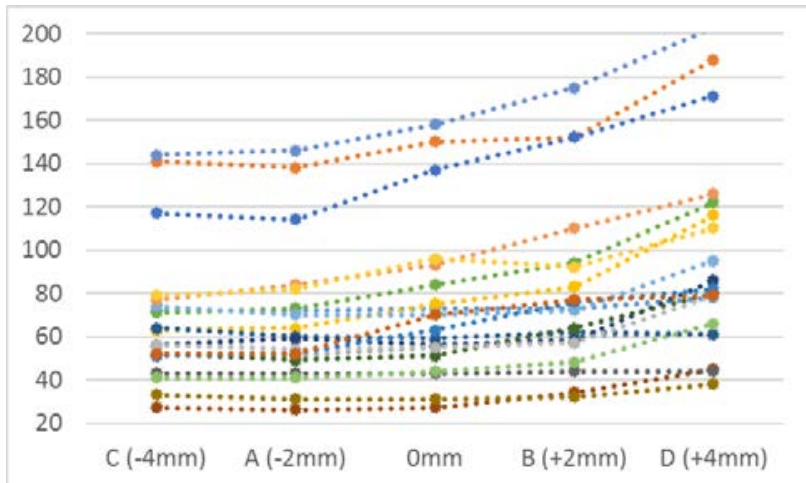

Fig. 11. Sample resistances (10\% glass phosphor by weight).

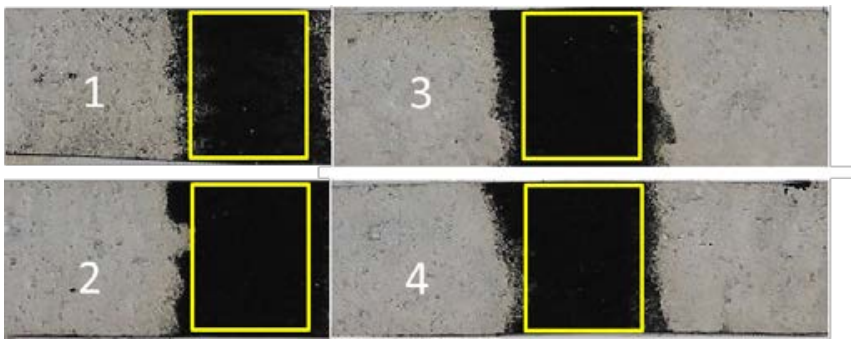

Fig. 12. Images of 4 samples (5\% glass phosphor)

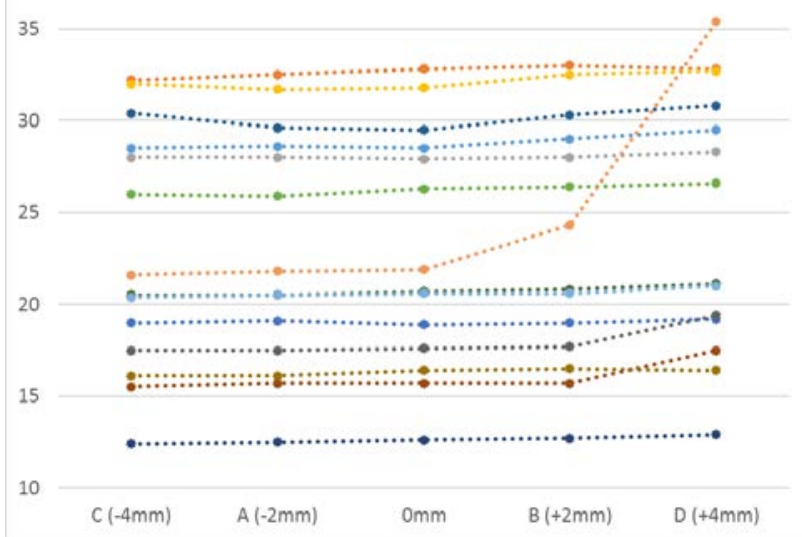

Fig. 13. Resistance of samples (5\% glass phosphor)
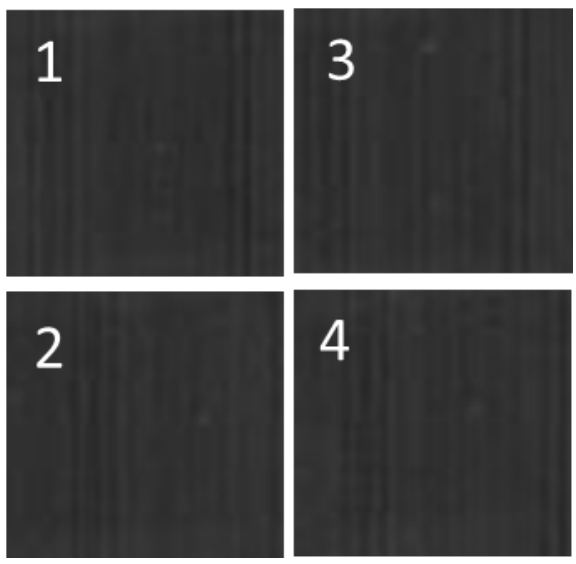

Fig. 14. Samples’ infrared images

shown in Fig. 14. Although the emission intensity is weak, the luminescence of the phosphor particles can be observed as whitish spots. There is a relationship between the density of the glass phosphor particles and the intensity of infrared light emission. Hence, the infrared light image can be used as an information feature. In the future, we will create a method to capture the luminescence from the particles more sharply.

\section{Conclusion}

In this paper, we proposed multimodal artifact metrics 


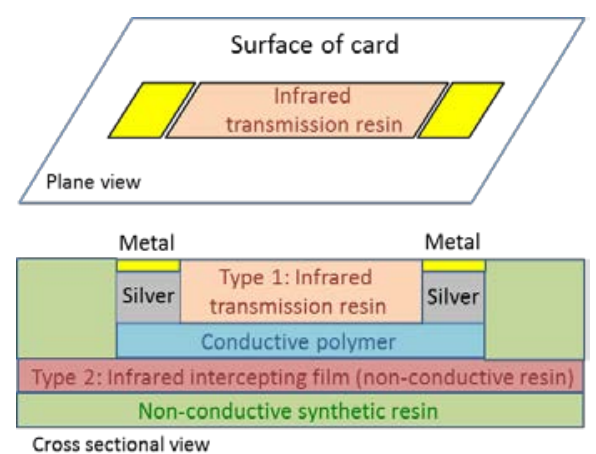

Fig. 15. Incorporation of thin film into valuable cards.

in order to authenticate an artifact with high accuracy and heighten the difficulty of counterfeiting. This technique is based on multimodal identification in biometrics. This method can embed two or more information features generated from two or more physical characteristics into an artifact and verify the authenticity of each artifact using the extracted information.

We focused on synthetic resin products as the artifacts for the application of multimodal artifact metrics. There are two types of synthetic resins, and in this paper, we used conductive synthetic resin (conductive polymer) and made samples by adding filler consisting of glass phosphor powder with optical characteristics. The result of our experiments shows that the two information features (electric resistance and infrared light image) extracted from each sample were different. This result indicates that our proposed method works well.

Samples made in the experiments are thin film and have flexibility, so we consider that it can heighten the difficulty of counterfeiting when they are implemented inside of valuable cards. In concrete, as shown in Fig. 15, we came up with the idea of sandwiching thin film between two types of non-conductive synthetic resin: type 1 has infrared light transmission function and type 2 has infrared light absorption feature (Hence, infrared cameras can capture the infrared light emitted only from glass phosphor particles). Next, terminals located in both side of thin film are exposed on the surface of the card and covered by hard metal (Hence, damages of thin film are prevented when probes are touched). In the future, we will make prototype and test its feasibility.

\section{Acknowledgment}

A part of this study was supported by the Kurata Memorial Hitach Science and Technology Foundation.

\section{References}

(1) ISO/IEC TR 24722:2015 : "Information technology -Biometrics -- Multimodal and other multibiometric fusion,” December 2015

(2) Arvind Slwal, Sunil Kumar Gupta, Surender, and Anubhuti : "Template security analysis of multimodal biometric frameworks based on fingerprint and hand geometry,” Perspectives in Science, Vol. 8, pp. 705/708, 2016

(3) Masaki Fujikawa, Fumihiko Oda, Kengo Moriyasu, Shingo Fuchi, and Yoshikazu Takeda : "Development of the New Artifact-metrics Technology for Valuable Pottery and Porcelain products," Journal of Information Processing Society of Japan, Vol. 55, No. 9, pp. 1992/2007, 2014

(4) Europol : "International credit card fraud syndicate active in Europe and Asia disrupted,” July 8th 2016, available: https://www.europol.europa.eu/content/inte rnational-credit-card-fraud-syndicate-active-europe-an d-asia-disrupted

(5) Focus Taiwan : "Taiwanese arrested for using counterfeit bank cards in Bangkok,” July 4th 2016, available:

http://focustaiwan.tw/news/asoc/201607040020.aspx

(6) CBS Miami : "Father, Son Arrested In \$50K Gift Card Fraud Raid,” September 1st 2016, Available: http://miami.cbslocal.com/2016/09/01/counterfeit-credi t-card-operation-busted-in-sw-dade/

(7) CASIO America Inc. : "Buyer Beware of Purchases Made From Unauthorized Resellers Of Casio premier G-Shock Products,” Available: http://www.gshock.com/support/Unauthorized

(8) news.com.au : "Tens of thousands of cunterfeit wheels made in China are on Australian roads, experts warn,” Available:

http://www.news.com.au/finance/business/manufacturi ng/tens-of-thousands-of-counterfeit-wheels-made-in-ch ina-are-on-australian-roads-experts-warn/news-story/b bf2565440a7ddf0c291a7ef9c4e9815

(9) Hiroshi Ishikawa and Yoshihiro Tomioka : "Deterioration of Polymer Material, Journal of the Society of Heating," Air-Conditioning and Sanitary Engineers of Japan, Vol. 79, No. 10, pp.961/968, 2005

(10) Akio Taniguchi : "Application of Organic Semiconductors,” CMC Technical Library, No. 297, pp. 100/101, CMC publishing. 